J. Lake Sci.(湖泊科学), 2008, 20(5): 655-661

http://www.jlakes.org. E-mail: jlakes@niglas.ac.cn

(C)2008 by Journal of Lake Sciences

\title{
基于泥沙因子的水深遥感反演模型*
}

\author{
间 峰 ${ }^{1}$, 王艳姣 ${ }^{2}$ \\ (1: 北京师范大学减灾与应急管理研究院, 北京 100875) \\ (2: 中国气象局国家气候中心, 北京 100081)
}

摘 要: 针对悬浮泥沙影响水体遥感测深精度的问题, 选择长江口南港至南槽为研究区, 通过对遥感测深方法研究, 结合悬 浮泥沙光谱特性分析，把“泥沙因子”引人到水体遥感测深反演模型中, 研究表明: 1)单因子非线性模型中, 指数模型对 $0-2 \mathrm{~m}$ 的水深反演效果较好, 对数模型对 $2-7 \mathrm{~m}$ 的水深反演较好, 二次回归模型对 7-14m 的水深反演效果较好; 2)建立的 BP 人工神 经网络水深反演模型综合了多个波段具有的水深信息, 模型的反演效果好于单因子非线性模型; 3)实验构建的泥沙遥感参数 综合了不同波段具有的悬沙信息，削弱了叶绿素和外界环境条件对泥沙信息的干扰，可较好地反映悬沙浓度变化特征；4)建 立的 BP 人工神经网络泥沙因子水深反演模型削弱了悬浮泥沙对遥感测深的影响, 模型实际反演能力明显优于单因子非线性 模型和多因子 BP 人工神经网络水深反演模型.

关键词: 水深遥感; 泥沙影响因子; 光谱反射率; 模型

\section{Water depth retrieval models with remote sensing sediment parameter}

\author{
YAN Feng $^{1} \&$ WANG Yanjiao ${ }^{2}$ \\ (1: Academy of Disaster Reduction and Emergency Management, Beijing Normal University, Beijing 100875, P.R.China) \\ (2: China Meteorological Administration, National Climate Center, Beijing 100081, P.R.China)
}

Abstract: In order to solve the problem of suspended sediment influencing water depth measurements by remote sensing technology, the remote sensing sediment parameter was constructed by experimental methods and introduced into the research of water depth retrieval methods to weaken the effect of suspended sediment to remote sensing bathymetry. The study area is the South Channel to the South Passage in the Yangtze River Estuary. Results showed that: 1) the exponential models can estimate the water depth of less than $2 \mathrm{~m}$ well, and the logarithm models can retrieve water depths from $2 \mathrm{~m}$ to $7 \mathrm{~m}$ accurately and the quadratic regression models can calculate the water depths from $7 \mathrm{~m}$ to $14 \mathrm{~m}$ better in the single factor nonlinear inversion models of water depth; 2) the multiple factor water depth inversion model of BP artificial neural network (BPANN) integrate water depth information of multiple wavelengths, and it performs the stronger capability to retrieve the water depths than single factor nonlinear models; 3)remote sensing sediment parameter constructed by experimental methods integrate suspended sediment information of multiple wavelengths, and weakens the effect of chlorophyll and environmental factors, and it can characterize the variations of suspended sediment concentration (SSC) better; 4) the sediment parameter water depth retrieval model of BP artificial neural network (SPBPANN) weakens the influence of SSC to remote sensing bathymetry, it performs the strongest capability than single factor nonlinear models and BPANN model.

Keywords: Remote sensing of water depth; sediment parameter; spectral reflectance; model

遥感测量水深主要是基于光在水体中的辐射传输原理. 太阳光通过大气和水体到达水底, 被水底反 射后又经水体和大气被卫星传感器接收, 通过提取出卫星传感器接收的水底反射光信息, 结合实际测量

* 国家博士后科学基金(20070420308)和淮河流域气象开放基金项目(HRM200605)联合资助. 2007-07-27 收稿; 2007-11-06 收修改稿. 闻峰, 男, 1973 年生, 博士; E-mail: njuyf@163.com. 
水深资料和一定的模型运算来获得大范围的水深情况. 自 20 世纪 70 年代起，国内外学者就在遥感探测 水深方面进行了研究 ${ }^{[1-3]}$, 并取得了一定的成果. 之后随着遥感技术的不断提高, 遥感测深技术得以迅速 发展, 逐渐形成了理论解译模型 ${ }^{[1]}$ 、半理论半经验模型 ${ }^{[2-6]}$ 和统计相关模型 ${ }^{[7-11]}$ 等多种水体遥感测深方法. 目前, 尽管国内外学者在水深遥感领域做了大量的研究, 提出了众多形式的模型, 但由于影响遥感测深 的因素较多(如水体悬浮物浓度、水体底质等), 故至今尚无统一的定量模式或可靠的模型参数, 水深遥感 的测量精度也未达到大、中比例尺常规测量的要求. 提高水深遥感解译的定量化水平, 必须去除或削弱 环境因素的影响, 特别是水质和水体底质对遥感测深的影响. 本文根据长江口水体浑浊度高的特点, 针 对悬浮泥沙影响水体遥感测深精度的问题, 选择长江口南港至南槽段为研究区, 通过对该区遥感测深方 法的研究, 结合悬浮泥沙光谱特性分析, 将悬浮泥沙的影响引入到遥感测深方法研究中, 旨在消除或削 弱悬沙对遥感测深的影响, 提高遥感测深方法的精度.

\section{1 研究区及资料处理}

选取长江口南港一南槽河段为研究区(图 1), 经纬度范围为 $121^{\circ} 40^{\prime} 40^{\prime \prime}-121^{\circ} 59^{\prime} 21^{\prime \prime} \mathrm{E}, 31^{\circ} 7^{\prime} 58^{\prime \prime}-31^{\circ} 21^{\prime} 47^{\prime \prime} \mathrm{N}$. 研究区水质和水体底质均匀且水深分布差异较大, 平均水深约为 $5.48 \mathrm{~m}$ (本文定义理论基准面以下为正值, 反之为负).

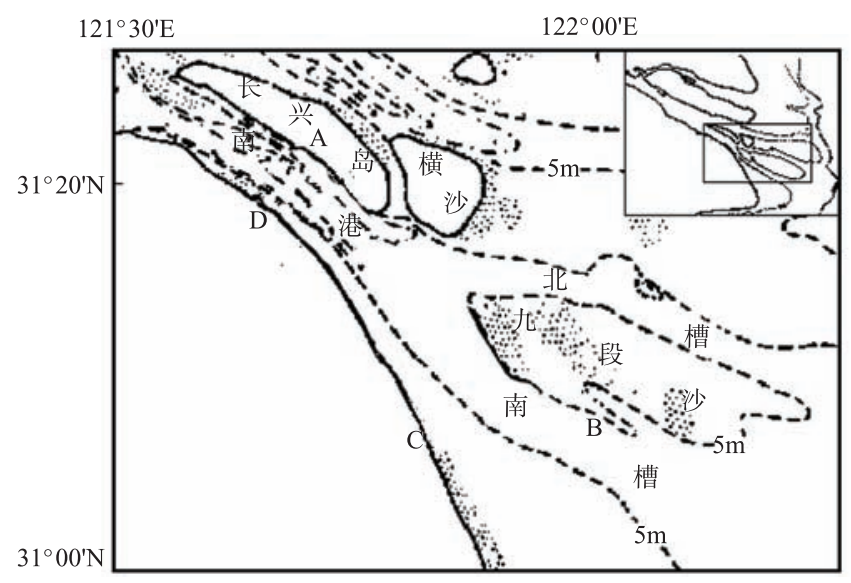

图 1 研究区示意图

Fig.1 Sketch of the study area

选取的图像资料为长江口 Landsat ETM+卫星遥感图像，成像时间为 2002 年 11 月 7 日. 采用改进后 的 DOS(dark object subtraction)法 ${ }^{[12]}$ 对图像进行了大气校正, 以长江口 1:10000 的实测地形图对遥感图像 进行几何精校正，并采用谱间分析法 $(T M 2+T M 3)>(T M 4+T M 5)$ 对图像进行了水陆分离 ${ }^{[13]}$.

所用水深资料为 2002 年 9 月实测的 1:25000 的水下地形图. 对收集的水下地形图进行扫描、几何配 准和数字化, 将其插值为与遥感图像具有相同空间分辨率的栅格图像 $(30 \mathrm{~m} \times 30 \mathrm{~m})$. 通过选取感兴趣区 (均 匀覆盖研究区的点), 选出相匹配像元反射率值和水深点 1310 个. 惖除那些通过掩膜后反射率为 0 的值 (由于海岸的变化引起的水深点和陆地相对应的值), 最终获得可利用的水深数据 1261 个.

\section{2 水深反演模型建立}

由于波段组合可综合多波段的水深信息，因此对各波段进行了多种组合运算. 将单波段、波段组合 值分别和水深值进行相关分析, 发现 $T M 4 、 T M 1 \cdot T M 4 、 T M 2 \cdot T M 4 、 T M 3 \cdot T M 4$ 和水深值的相关性较好, 相 关系数绝对值均在 0.8 以上(表 1). 研究表明, 可见光波段对水体的穿透性较强(TM1-TM3), 而近红外波 段(TM4)对水体的穿透性较弱. 在水质较清的河流和湖泊, 遥感测深主要是基于水底对可见光的反射来 
获得水深信息 ${ }^{[11,14-15]}$, 而在水体相对混浊的河口和海岸，水体对光波的后向散射将大于水底对光波的反 射，水体后向散射分量与水体深度具有良好的相关关系，具此也可以较好的估算水深 ${ }^{[16-18]}$. 由于长江口

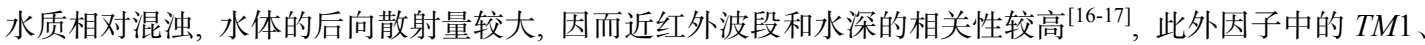
$T M 2$ 和 $T M 3$ 项又具有一定水底对光波的反射信息. 可见，本文选取的水深反演因子综合了水底和水体反 射的水深信息，因而可较好的反演研究区水深分布情况.

表 1 各波段和波段组合与相应水深值的相关系数

Tab.1 Correlation coefficients between the bands, band combinations and water depth values

\begin{tabular}{lcccc}
\hline 因子 & $T M 4$ & $T M 1 \cdot T M 4$ & $T M 2 \cdot T M 4$ & $T M 3 \cdot T M 4$ \\
\hline 相关值 & -0.84 & -0.82 & -0.82 & -0.83 \\
\hline
\end{tabular}

\section{1 非线性水深反演模型}

光波在水体中随着水深的变化呈指数形式衰减, 即水体中光辐射传输过程是一个非线性过程. 为了 较好地反演水深, 采用选取的 1261 个相对应的水深点和水深因子分别建立了指数、对数和二次回归方程 等多种模型(表 2). 所建方程均超过 $a=0.01$ 的 $F$ 信度检验，可以作为水深反演模型.

为了较好的检验模型精度和模型的稳定性, 利用在研究区均匀选点感兴趣区的方法从水深图和遥感 图像中随机取出两组数据, 第一组为 514 个相对应的水深点和像元反射率值, 第二组为 503 个相对应的 点. 由于这两组数据没有参加建模, 因此可将其看作是不受模型影响的独立检验样本. 分别将这两组数 据代人模型，计算了研究区水深. 为了定量的比较各模型的反演效果，将水深值按深度分为三段，计算 了各水深段平均相对误差以及整体水深误差(表 2), 发现利用各因子建立的不同模型对不同段水深反演 情况不同, 其中指数模型对 $0-2 \mathrm{~m}$ 的水深情况反演较好, 对数模型对 $2-7 \mathrm{~m}$ 的水深情况反演较好, 而二次 回归模型对 $7-14 \mathrm{~m}$ 的水深反演较好. 三种模型中以对数模型对整体的水深范围 $(0-14 \mathrm{~m})$ 反演效果最好, 其中 TM4 的对数模型对研究区水深反演精度最高, 模型反演值和实测值之间的平均相对误差分别为 $19.69 \%$ 和 $18.42 \%$, 均优于其它模型.

表 2 单因子非线性模型各水深段平均相对误差的比较

Tab.2 Comparison of mean relative errors of different water depths retrieved by single factor nonlinear models

\begin{tabular}{|c|c|c|c|c|c|c|c|c|c|}
\hline \multirow{2}{*}{ 因子 } & \multirow{2}{*}{ 模型类型 } & \multicolumn{4}{|c|}{ 平均相对误差 (1 组) } & \multicolumn{4}{|c|}{ 平均相对误差(2 组) } \\
\hline & & $0-2 \mathrm{~m}$ & $2-7 \mathrm{~m}$ & $7-14 m$ & $0-14 \mathrm{~m}$ & $0-2 \mathrm{~m}$ & $2-7 \mathrm{~m}$ & $7-14 \mathrm{~m}$ & $0-14 \mathrm{~m}$ \\
\hline \multirow{3}{*}{ TM4 } & $Y=0.00007 x^{2}-0.0933 x+17.614$ & 0.6251 & 0.5467 & 0.1045 & 0.3276 & 0.5681 & 0.4706 & 0.1254 & 0.2938 \\
\hline & $Y=62.679 \mathrm{e}^{-0.0225 x}$ & 0.3065 & 0.2704 & 0.1949 & 0.2245 & 0.2858 & 0.2803 & 0.2140 & 0.2418 \\
\hline & $Y=-11.469 \ln (x)+60.05$ & 0.5561 & 0.2645 & 0.1358 & 0.1969 & 0.2597 & 0.2360 & 0.1459 & 0.1842 \\
\hline \multirow{3}{*}{$T M 1 \cdot T M 4$} & $Y=8 . \mathrm{E}-09 x^{2}-0.0006 x+11.637$ & 0.5849 & 0.4552 & 0.1328 & 0.2581 & 0.5132 & 0.3856 & 0.1243 & 0.2375 \\
\hline & $Y=12.781 \mathrm{e}^{-0.00008 \mathrm{x}}$ & 0.4232 & 0.4141 & 0.1544 & 0.2508 & 0.4418 & 0.3741 & 0.1480 & 0.2444 \\
\hline & $Y=-3.821 \ln (x)+41.28$ & 0.3431 & 0.4017 & 0.1576 & 0.2451 & 0.3748 & 0.3241 & 0.1576 & 0.2286 \\
\hline \multirow{3}{*}{$T M 2 \cdot T M 4$} & $Y=2 . \mathrm{E}-09 x^{2}-0.0004 x+12.328$ & 0.5509 & 0.4606 & 0.1246 & 0.2530 & 0.5629 & 0.3637 & 0.1233 & 0.2303 \\
\hline & $Y=16.383 \mathrm{e}^{-0.00007 x}$ & 0.3592 & 0.4292 & 0.1722 & 0.2639 & 0.3887 & 0.3781 & 0.1751 & 0.2598 \\
\hline & $Y=-4.889 \ln (x)+52.939$ & 0.5644 & 0.3946 & 0.1706 & 0.2613 & 0.4422 & 0.3255 & 0.1780 & 0.2435 \\
\hline \multirow{3}{*}{$T M 3 \cdot T M 4$} & $Y=7 . \mathrm{E}-09 x^{2}-0.0007 x+16.023$ & 0.5525 & 0.4400 & 0.1449 & 0.2592 & 0.7753 & 0.4368 & 0.1542 & 0.2837 \\
\hline & $Y=22.353 \mathrm{e}^{-0.00007 x}$ & 0.3641 & 0.3644 & 0.1443 & 0.2256 & 0.3778 & 0.3432 & 0.1703 & 0.2434 \\
\hline & $Y=-6.763 \ln (x)+72.623$ & 0.4227 & 0.3453 & 0.1313 & 0.2140 & 0.4336 & 0.3231 & 0.1554 & 0.2290 \\
\hline
\end{tabular}

\subsection{BP 人工神经网络模型(BPANN)}

为了提高模型对研究区水深的反演精度, 尝试选用多因子来建立模型. 采用逐步回归方法 $(F$ 值取 0.01)对 2.1 节方程中的非线性项进行篮选，最终选取和水深信息显著相关的 $T M 4^{2} 、 \mathrm{e}^{-0.0225 \cdot T M 4} 、 \ln (T M 4)$ 、 
$\mathrm{e}^{-0.00008 \cdot T M 1 \cdot T M 4}$ 和 $\ln (T M 3 \cdot T M 4)$ 作为水深反演因子.

$\mathrm{BP}$ 人工神经网络是一种大规模并行的非线性动态系统, 具有较强的模拟非线性动态变化的能力, 利 用上面选取的非线性水深因子，建立了一个 3 层的人工神经网络模型，其中隐层神经元采用的是 Tansig 传递函数, 输出层采用的是 Purelin 函数. 本文在原有 BP 网络的基础上，针对 BP 网络易陷人局部最小值、 收玫速度慢和过拟合等缺陷，通过加人动量项和引人权值控制算法对网络进行了改进，具体方法参见文 献[19-20]. 利用前面选取的非线性因子作为网络模型的学习输人样本，相应的水深值作为学习矩阵的期 望输出. 为了和单因子非线性模型进行比较, 同样选取 1261 个数据建立模型, 利用两组数据来检验模型. 通过对输人序列和输出序列之间对应关系的反复学习和训练, 来确定网络模型的隐层节点数和其他可调 参数,并不断调整网络模型输人层与隐层、隐层和输出层之间的连接权值和域值, 找出其传递函数的最佳 权值矩阵和域值矩阵，从而建立遥感反射率和水深值之间的映射关系. 通过实验，最终确定 BP 网络模型 的结构为: 输人层节点数取 5 , 隐含层节点数取 18 , 输出层节点数取 1 ; 各参数值分别确定为: 学习速率 取 0.01 , 学习速率增加和减少比率取 1.2 , 动量参数取 0.95 , 训练精度取 0.5 , 误差比率取 0.5 .

为了对 BP 网络模型进行检验, 将两组检验数据代人模型, 计算了各水深段平均相对误差和整体水深 误差(表 3). 由表 3 可见模型对整体水深反演效果较好，实测水深值和估算值之间的总体平均相对误差分 别为 $16.75 \%$ 和 $17.01 \%$, 反演效果好于单因子非线性模型. 对水深进行分段比较表明, BP 人工神经网络模 型对 0-2m 的水深其平均相对误差在 30\%以内，对 2-7m 的水深其平均相对误差在 $25 \%$ 以内，而对 7-14m 的水深平均相对误差均小于 $10 \%$. 可见, BP 人工神经网络水深反演模型对不同段水深反演精度均有一定 的提高.

以上对遥感测深方法研究表明, 由于遥感测深受多种环境因素的影响(如水质和水体底质等), 算法 上的改进不能很好的提高遥感测深的精度, 若要进一步提高其反演精度, 必须削弱外界因素对遥感测深 的影响.

表 3 多因子 BP 人工神经网络模型和 BP 泥沙因子各水深段平均相对误差

Tab.3 Mean relative errors of different water depths retrieved by BPANN model and SPBPANN model

\begin{tabular}{cccccc}
\hline \multirow{2}{*}{ 水深 $(\mathrm{m})$} & \multicolumn{2}{c}{ BPANN 模型 } & & \multicolumn{2}{c}{ SPBPANN 模型 } \\
\cline { 2 - 3 } \cline { 5 - 6 } & 1 组检验数据 & 2 组检验数据 & & 1 组检验数据 & 2 组检验数据 \\
\hline $0-2$ & 0.2987 & 0.3149 & & 0.2406 & 0.2471 \\
$2-7$ & 0.2352 & 0.2392 & & 0.1969 & 0.1907 \\
$7-14$ & 0.0923 & 0.0958 & & 0.0805 & 0.0836 \\
$0-14$ & 0.1675 & 0.1701 & & 0.1382 & 0.1452 \\
\hline
\end{tabular}

\section{3 泥沙因子水深反演模型}

\section{1 悬沙浓度敏感因子的选取}

为了获得悬沙浓度敏感因子, 本文进行了不同浓度悬沙水体配比试验 ${ }^{[21]}$. 通过分析不同浓度悬沙水 体光谱特征、光谱反射率和悬沙浓度的相关性，确定悬浮泥沙敏感波段范围为 $r_{1}(430-500 \mathrm{~nm})$ 、 $r_{2}(550-570 \mathrm{~nm}) 、 r_{3}(670-735 \mathrm{~nm})$ 和 $r_{4}(780-835 \mathrm{~nm})$. 分析这些敏感波段平均值 $R_{n}\left(R_{1}, R_{2}, R_{3}\right.$ 和 $\left.R_{4}\right)$ 及其波段 比值与悬沙浓度之间的多种关系模型(线性、二次和三次回归), 选取能较好反映悬沙浓度变化的 $R_{n}$ 及其 波段比值构建了泥沙遥感参数 $S_{p}^{[21]}$ :

$$
S_{p}=\frac{\left(R_{2}+R_{3}\right)}{R_{1} / R_{2}}
$$

式中, $R_{1} 、 R_{2}$ 和 $R_{3}$ 分别代表各波段范围反射率的平均值. 已有的研究表明 $490 \mathrm{~nm} 、 550 \mathrm{~nm}$ 和 $670 \mathrm{~nm}$ 波段 附近是悬沙浓度的敏感波段，而 $490 \mathrm{~nm}$ 和 $550 \mathrm{~nm}$ 波段又分别位于叶绿素的吸收峰和反射峰上，这两个波 段附近对叶绿素也比较敏感 ${ }^{[22-23]}$. 因此, 在泥沙遥感参数中, $\left(R_{2}+R_{3}\right)$ 能较好反映低悬沙浓度的变化特征, $R_{1} / R_{2}$ 可去除叶绿素对悬浮泥沙的干扰. 此外, 参数中采用比值算法可去除环境因素对光谱反射率的影响. 
图 2 为利用实验光谱数据计算的泥沙遥感参数与实测悬沙浓度对数的比较, 可以看出泥沙遥感参数和实测 悬沙浓度对数变化较为一致, 可见本文建立的泥沙遥感参数能较好的反映悬沙浓度变化特征.

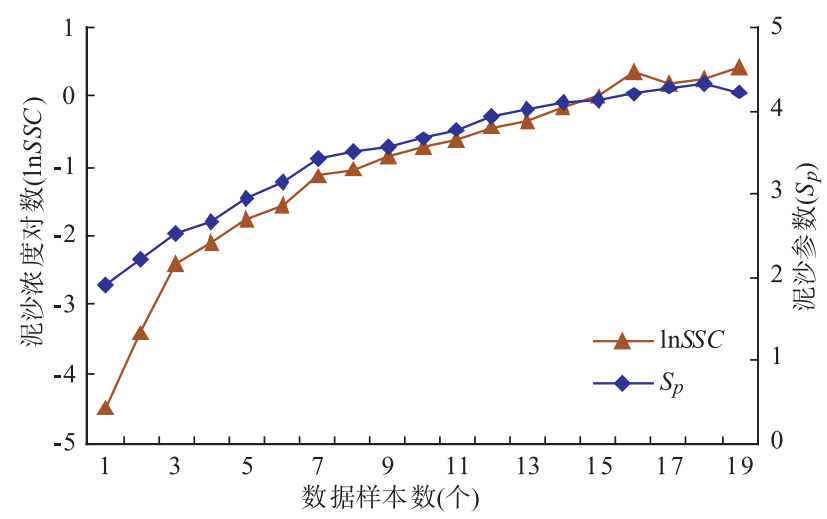

图 2 实测悬沙浓度对数和泥沙遥感参数比较

Fig.2 Comparison of SSC logarithms and sediment parameter

\section{2 悬沙浓度影响因子的确定}

长江口是我国较大的内陆河口, 水体较浑浊, 表层悬沙含量是影响该区遥感测深的一个重要的因子. 由于遥感测深是通过光在水体内的辐射衰减来进行, 当表层悬沙浓度发生变化时, 将使水体内光的辐射 衰减发生变化而影响遥感测深精度.

3.1 中的研究表明, 泥沙遥感参数 $S_{p}$ 具有较多的泥沙信息且能很好的反演悬沙浓度的变化, 因而选取 $S_{p}$ 作为遥感测深的悬沙影响因子. 在泥沙遥感参数中, $R_{1}(430-500 \mathrm{~nm}) 、 R_{2}(550-570 \mathrm{~nm})$ 和 $R_{3}(680-735 \mathrm{~nm})$ 分别对应 ETM+遥感影像的 $T M 1(450-520 \mathrm{~nm}) 、 T M 2(520-600 \mathrm{~nm})$ 和 $T M 3(630-690 \mathrm{~nm})$ ，因而可利用下式计 算 ETM+遥感影像的泥沙遥感参数:

$$
S_{P}=\frac{(T M 2+T M 3)}{T M 1 / T M 3}
$$

为了检验该泥沙遥感参数所具有的信息, 将 $\mathrm{ETM}+$ 影像 4 个波段的像元反射率分别与 $\ln S_{p}$ 进行了比 值运算, 并计算了各比值和实测水深之间的相关性. 结果表明, 当 4 个波段和 $\ln S_{p}$ 进行比值运算后，它们 和实测水深值之间的相关性都有了一定的提高, 其中以 $T M 2 / \ln S_{p}$ 和 $T M 3 / / \mathrm{ln} S_{p}$ 相关性提高较多. 由于 $T M 2(520-600 \mathrm{~nm})$ 和 $T M 3(630-690 \mathrm{~nm})$ 正好位于悬沙水体光谱反射峰上 ${ }^{[21]}$, 即在这两个波段处，悬沙浓度 变化对光谱反射率影响较大, 当将这两个波段处像元反射率和 $\ln S_{p}$ 进行比值运算后, 削弱了悬沙浓度对 像元反射率的影响，提高了它们和实测水深之间的相关性.

\subsection{BP 泥沙因子水深反演模型(SPBPANN)}

利用 3.2 中选出的较好的非线性水深反演因子 $T M 4^{2} 、 \mathrm{e}^{-0.0225 \cdot T M 4} 、 \ln (T M 4) 、 \mathrm{e}^{-0.00008 \cdot T M 1 \cdot T M 4}$ 和 $\ln (T M 3 \cdot T M 4)$, 将这些因子中所含各波段及波段组合和 $\ln S_{R}$ 进行了比值运算. 表 4 列出了比值运算后这些 因子和三组实测水深之间相关性的比较，当各波段及波段组合和 $\ln S_{p}$ 进行比值运算后，它们和实测水深 值之间的相关性都有了一定的提高，即本文引入的 $\ln S_{p}$ 项削弱了悬浮泥沙对各波段和波段组合像元反射 率的影响, 提高了它们和实测水深之间的相关性(表 4). 这里将 $\ln S_{p}$ 称为“泥沙影响因子”.

利用表 4 中已削弱泥沙影响的波段及波段组合作为水深反演因子，重新建立了 BP 人工神经网络泥沙 因子水深反演模型, 其结构为: 输人层节点数取 5 , 隐含层节点数取 20 , 输出层节点数取 1 ; 各参数值分 别确定为: 学习速率取 0.01 , 学习速率增加和减少比率取 1.0 , 动量参数取 0.90 , 训练精度取 0.5 , 误差比 率取 0.65 . 
表 4 各波段及波段组合和 $\ln S_{R}$ 比值前后与实测水深之间相关性比较

Tab.4 Correlation comparison between the radios of each band, band combinations and $\ln S_{p}$ and measured water depth values

\begin{tabular}{lccc}
\hline 波段及波段组合 & 建模水深数据 & 1 组检验水深数据 & 2 组检验水深数据 \\
\hline$T M 4^{2}$ & -0.84 & -0.78 & -0.80 \\
$T M 4^{2} / \ln S_{p}$ & -0.89 & -0.84 & -0.85 \\
$T M 1 \cdot T M 4$ & -0.82 & -0.70 & -0.72 \\
$(T M 1 \cdot T M 4) / \ln S_{p}$ & -0.89 & -0.80 & -0.81 \\
$T M 3 \cdot T M 4$ & -0.83 & -0.78 & -0.79 \\
$(T M 3 \cdot T M 4) / \ln S_{p}$ & -0.93 & -0.89 & -0.90 \\
\hline
\end{tabular}

为了和 3.2 节中建立的模型进行比较, 同样将两组检验数据代人模型, 计算了各水深段平均相对误 差和整体水深误差. SPBPANN 模型对整体水深反演误差分别为 $13.82 \%$ 和 $14.52 \%$, 反演精度比 BPANN 模 型有了较大的提高(表 3). 比较两个模型对不同段水深反演效果发现, SPBPANN 模型对 0-2m 的水深其平 均相对误差在 $25 \%$ 以内, 对 2-7m 的水深其平均相对误差在 $20 \%$ 以内, 而对 7-14m 的水深平均相对误差 均小于 $8.5 \%$, 即 SPBPANN 模型对不同段水深反演精度比 BPANN 模型均有较大的提高. 可见，由于引人 了“泥沙影响因子”, 削弱了悬沙浓度对遥感测深的影响, 因而 SPBPANN 模型的反演效果好于 BPANN 模 型.

\section{4 结论}

目前, 虽然国内外学者分别在水深遥感和悬浮泥沙光谱特性分析方面做了大量的研究, 但在水深遥 感方法研究中考虑悬浮泥沙影响方面, 国内外还未见研究发表. 本文针对悬浮泥沙影响水体遥感测深精 度的问题, 选择长江口南港一南槽为研究区, 探讨了遥感测深方法中消弱悬浮泥沙影响的方法, 主要结 论如下:

(1) 不同单因子非线性模型对不同段水深反演情况不同. 指数模型对 0-2m 的水深反演效果较好, 对 数模型对 $2-7 \mathrm{~m}$ 的水深反演较好, 而二次回归模型对 7-14m 的水深反演效果较好. 在各单因子非线性模 型中, 以 TM4 的对数模型对研究区整体的水深范围 $(0-14 \mathrm{~m})$ 反演效果最好.

(2) 建立了多因子 BP 人工神经网络水深反演模型. 由于该模型综合了多个波段所具有的水深信息, 其反演效果好于单因子非线性模型.

(3) 本文构建的泥沙遥感参数，既综合了不同波段具有的悬沙信息，也削弱叶绿素和外界环境条件 对泥沙信息的干扰，因而能很好的反映悬沙浓度的变化特征.

(4) 建立了 BP 人工神经网络泥沙因子水深反演模型. 由于模型中引人了“泥沙影响因子”, 削弱了悬 浮泥沙对遥感测深的影响, 模型精度有了较大的提高, 模型实际水深反演能力明显优于以上建立的单因 子非线性模型和多因子 BP 人工神经网络水深反演模型.

\section{5 参考文献}

[1] Lyzenga DR. Shallow-water reflectance modeling with applications to remote sensing of ocean floor. Proceeding of 13th International Symposium on Remote Sensing of Environment, Ann Arbor, Michigan, 1979: 583-602.

[2] Polcyn FC, Sattinger IJ. Water depth determination using remote sensing techniques. Proceedings of the 6th International Symposium on Remote Sensing of Environment, Ann Arbor, Michigan, 1969: 1017-1028.

[3] Polcyn FC, Lyzenga DR. Calculation of water depth from ERTS-MSS data. Proceedings Symposium on Significant Results Obtained from ERTS-1, New Carrollton, Maryland, 1973: 1433-1436.

[4] Tanis FJ, Hallada WA. Evaluation of Landsat Thematic Mapper data for shallow water bathymetry. Proceeding of 18th International Symposium on Remote Sensing of Environment, Ann Arbor, Michigan, 1984: 629-643. 
[5] Tanis FJ, Byrne HJ. Optimization of multispectral sensors for bathymetry applications. Proceeding of 19th International Symposium on Remote Sensing of Environment, Ann Arbor, Michigan, 1985: 865-874.

[6] Wei J, Daniel LC, William CK. Satellite remote bathymetry: a new mechanism for modeling. Photogrammetric Engineering and Remote Sensing, 1992, 58(5): 545-549.

[7] Hengle WV, Spitzer D. Multi-temporal water depth mapping by means of Landsat TM. International Journal of Remote Sensing, 1991, 12(4): 703-712.

[8] Baban SM. The evaluation of different algorithms for bathymetric charting of Lakes using Landsat imagery. International Journal of Remote Sensing, 1993, 14(12): 2263-2273.

[9] Sandidge JC, Holyer RJ. Coastal bathymetry from hyperspectral observations of water radiance. Remote Sensing of Environment, 1998, 65: 341-352.

[10] Tripathi NK, Rao AM. Bathymetric mapping in Kakinada Bay, India, using RS-1D LISS-III data. International Journal of Remote Sensing, 2002, 23(6): 1013-1025.

[11] 于瑞宏, 刘廷玺, 李畅游等. 干旱区草型湖泊悬浮固体浓度及水深的遥感与分析. 水利学报, 2005, 36(7): 853-862.

[12] Mausel PD, Brondizio E, Moran E. Assessment of atmospheric correction methods for Landsat TM data applicable to amazon basin LBA reaearch. International Journal of Remote Sensing, 2002, 23(13): 2651-2671.

[13] 周成虎, 骆剑承, 杨晓梅等. 遥感影像地学理解与分析. 北京: 科学出版社, 2001: 75-78.

[14] 邸凯昌, 丁 谦, 陈 薇等. 南沙群岛海域浅海水深提取及影像海图制作技术. 国土资源遥感, 1999, (3): 59-64.

[15] 沈 芳, 匡定波. 青海湖最近25年变化的遥感调查与研究. 湖泊科学, 2003, 15(4): 289-296.

[16] 张 东, 王 文, 张 鹰. 长江口水域水深遥感研究. 河海大学学报(自然科学版), 1998, 26(6): 85-90.

[17] 黄家柱, 尤玉明. 长江南通河段卫星遥感水深探测试验. 水利科学进展, 2002, 13(2): 235-238.

[18] 田庆久, 王晶晶，杜心栋. 江苏近海岸水深遥感研究. 遥感学报, 2007, 11(3): 373-379.

[19] 袁曾任. 人工神经网络及其应用. 北京: 清华大学出版社, 2000: 65-69.

[20] 张 翔, 丁 晶. 提高多层前馈网络推广能力的权值控制算法. 水科学进展, 1998, 4(9): 373-377.

[21] Wang YJ, Yan F, Zhang PQ et al. Experimental research on quantitative inversion models of suspended sediment concentrations using remote sensing technology. Chinese Geographical Science, 2007, 17(3): 243-249.

[22] 李四海, 唐军武, 恽才兴. 河口悬浮泥沙浓度SeaWiFS遥感定量模式研究. 海洋学报, 2002, 24(2): 51-58.

[23] Tassn S. Local algorithms using SeaWiFs data for the retrieval of phytoplankton, pigments, suspended sediment and yellow substance in coastal waters. Applied Optics, 1994, 33(12): 2369-2378. 